\title{
THE NEED FOR GROUND-BASED OBSERVATIONS OF VENUS
}

\author{
Authors: Candace Gray ${ }^{1}$ and Eliot Young ${ }^{2}$ \\ ${ }^{1}$ New Mexico State University - Apache Point Observatory, candanceg@nmsu.edu, \\ 575-646-6399 \\ ${ }^{2}$ Southwest Research Institute, Department of Space Studies, efy@boulder.swri.edu
} 303-546-6807

Co-Authors: Toru Kouyama (National Institute of Advanced Industrial Science and Technology), Yeon Joo Lee (Technical University of Berlin), Arnaud Mahieux (University of Texas at Austin), Emmanuel Marcq (Laboratoire Atmosphères, Milieux, Observations Spatiales (LATMOS)), Kevin McGouldrick (Laboratory for Atmospheric and Space Physics (LASP) at the University of Colorado Boulder), Thomas Navarro (UCLA), Glenn Orton (NASA/JPL), Javier Peralta (Japan Aerospace Exploration Agency), Emilie Royer (Planetary Science Institute), Kunio Sayanagi (Hampton University), Brad Sandor (Space Science Institute), Thomas Widemann (Laboratory of Space and Instrumentation Studies (LESIA)), Takeshi Imamura (University of Tokyo) 


\title{
THE NEED FOR GROUND-BASED OBSERVATIONS OF VENUS
}

\author{
Authors: Candace Gray ${ }^{1}$ and Eliot Young ${ }^{2}$ \\ Co-Authors: Toru Kouyama, Yeon Joo Lee, Arnaud Mahieux, Emmanuel Marcq, Kevin \\ McGouldrick, Thomas Navarro, Glenn Orton, Javier Peralta, Emilie Royer, Kunio Sayanagi, \\ Brad Sandor, Thomas Widemann, Takeshi Imamura \\ ${ }^{1}$ New Mexico State University - Apache Point Observatory, candanceg@nmsu.edu \\ ${ }^{2}$ Southwest Research Institute, Department of Space Studies, efy@boulder.swri.edu
}

\begin{abstract}
Ground-based observations of Venus are crucial to NASA's goals. They can complement spacecraft observations (for example, by obtaining additional spectral coverage or extended time baselines). Ground-based and spacecraft observations raise new questions and help direct the designs of new mission proposals. Finally, ground-based observations can make new discoveries and achieve cutting-edge science in and of themselves. In this white paper we review ground-based observations of Venus in the context of recent spacecraft results and key unanswered questions. The purpose of this white paper is to state the cases for supporting ground-based observations (some of which face stiff competition on facilities like ALMA), for necessary lab work (like measuring absorption coefficients under relevant conditions) and for improvements to ground-based observing infrastructure, notably adaptive optics (AO) systems for extended objects and mid-IR cameras to image Venus at 5 - $20 \mu \mathrm{m}$.
\end{abstract}

\section{INTRODUCTION}

Venus's atmosphere is recognized as a place to study coupled systems: atmospheric chemistry, dynamics, energy deposition and cloud microphysics are interconnected systems that produce a host of unique and observable phenomena. The previous two missions to Venus, Venus Express (ESA) and Akatsuki (JAXA) shared a common goal: to observe Venus's circulation and explain the origins of Venus's super-rotating atmosphere. While both missions provided cloud-tracking results that help constrain GCMs, their most important legacies lie elsewhere. Venus Express observations through atmospheric windows near $\sim 1 \mu \mathrm{m}$ produced maps of surface thermal emissivity anomalies, a result that influenced the design and science objectives of the VERITAS mission. Akatsuki's 10- $\mu \mathrm{m}$ LIR camera produces thermal maps (from $\sim 66 \mathrm{~km}$ altitude), showing stationary features caused by mountain waves that are launched from four large highland regions on the surface. Fourier decomposition of LIR disk temperatures reveal distinct planetary scale waves with periods near 3.9 and 5.3 days.

Both the Venus Express and Akatsuki missions illustrate how strong observing programs can lead to new, unexpected areas of exploration. Ground-based observing programs have played a similar role, and have added value to the Venus Express and Akatsuki data sets. Some examples: 
- Doppler winds derived from very high resolution visible spectroscopy confirmed that dayside cloud tracking can be used to construct wind fields (Machado et al. 2017).

- Submm and near-IR spectra were used to map $\mathrm{SO}_{2}$ and monitor its variability.

- Ground-based imaging of Venus's middle and lower cloud decks gave pole-to-pole coverage with resolutions of $150 \mathrm{~km}$ on Venus, extending Venus Express cloud tracking coverage of Venus's southern hemisphere.

- Ground-based spectroscopy from instruments like TripleSpec (APO), IRIS2 (AAO) and iSHELL (IRTF) improved upon the spectral resolution of Venus Express (Akatsuki has no spectroscopy) and helped map trace gases below Venus's cloud base.

The rest of this white paper covers some of the key science questions that can be addressed with ground-based imaging $(\S 2)$ and advocates specific infrastructure upgrades $(\S 3)$.

\section{GROUND-BASED OBSERVATIONS OF VENUS AND KEY QUESTIONS}

\subsection{DAY AND NIGHT CLOUD TRACKING}

The species in the Venus atmosphere that produce brightness contrasts (whether in dayside reflected solar light or in nightside thermal emission) are clouds and trace gases. The clouds are largely composed of sulfuric acid: their very low vapor pressure can drive rapid and substantial changes in the vapor/liquid equilibrium in the clouds. The clouds of Venus cannot be considered purely passive tracers - observed changes can be attributed to dynamical motions as well as chemistry and microphysics. Dayside cloud tracking in UV channels (283 and $365 \mathrm{~nm}$ ) provides wind fields at the cloud tops with sensitivity to $\mathrm{SO}_{2}$ and the unknown $\mathrm{UV}$ absorber, dayside tracking at $\sim 900 \mathrm{~nm}$ probes just below the cloud tops (around $66 \mathrm{~km}$ ), and nightside cloud tracking at 1.74 and $2.25-2.5 \mu \mathrm{m}$ probes the lower and middle cloud decks $(\sim 48-58 \mathrm{~km})$.
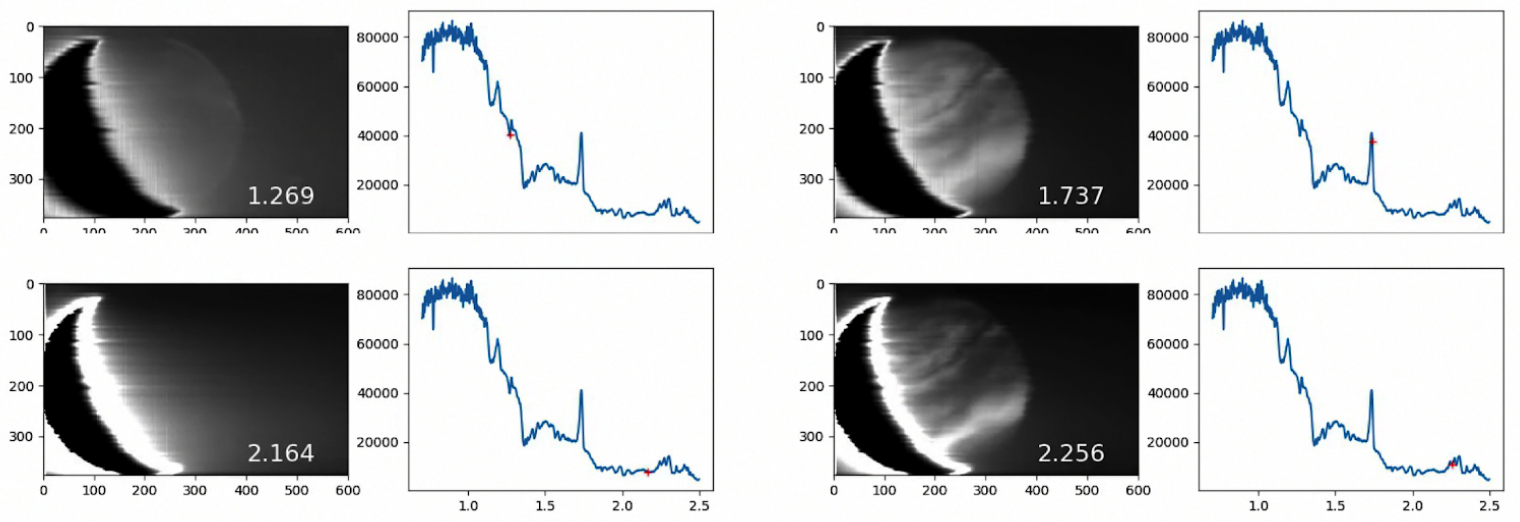

Fig. 1. Venus's nightside in four wavelengths (slices of a Venus spectral image cube obtained from the IRTF in April 2017) shows the transparency in $\mathrm{CO}_{2}$ windows. The dayside crescent is saturated. Top left: $1.27 \mu \mathrm{m}$ is a $\mathrm{CO}_{2}$ window. Venus's nightside disk is visible, plus $\mathrm{O}_{2}{ }^{1} \Delta$ emission (most visible over the nightside limb, originating from $95 \mathrm{~km}$ altitude). Top right: At $1.74 \mu \mathrm{m}\left(\mathrm{a} \mathrm{CO} \mathrm{C}_{2}\right.$ 
window), clouds in Venus's middle and lower cloud decks show up as silhouettes on Venus's night hemisphere. Bottom left: $2.16 \mu \mathrm{m}$ is not a $\mathrm{CO}_{2}$ window, so we see only scattered light from the saturated dayside crescent- no nightside disk is visible. Bottom right: Many species (CO, OCS, $\mathrm{H}_{2} \mathrm{O}, \mathrm{SO}_{2}$ ) have diagnostic absorptions within this broad $\mathrm{CO}_{2}$ window $(2.2-2.5 \mu \mathrm{m})$, and cloud properties can be determined using comparisons to the $1.74 \mu \mathrm{m}$ images. (Link to a movie showing Venus at $\lambda=0.8-2.45 \mu \mathrm{m}$ : <https://www.boulder.swri.edu/ efy/Trans20/gdMovie.mp4>).

Both spacecraft and ground-based imagers typically derive wind fields from image sequences that span an hour or more. Cross-correlations between small subframes provide offsets and velocities (after dividing by the time interval between frames). Ground-based image sequences can produce wind fields with errors less than $3 \mathrm{~m} / \mathrm{s}$ as follows. Cross-correlations between 400-km subframes typically yield offsets with $30 \mathrm{~km}$ errors (rms). Over a four-hour observing window, the $30 \mathrm{~km}$ error translates into a velocity error of $2.1 \mathrm{~m} / \mathrm{s}$. It is important to get the velocity errors down to $\sim 1 \mathrm{~m} / \mathrm{s}$ in order to resolve the meridional motions predicted by Horinouchi et al. (2020). Low errors can be achieved with a network of telescopes spanning longitudes around the Earth. For example, the Nordic Optical Telescope and the IRTF are separated by 10 hours in longitude. Velocities derived from those two sites using Advection Corrected Correlation Image Velocimetry (Asay-Davies et al., 2009) have errors under $1 \mathrm{~m} / \mathrm{s}$.

\subsection{SUBMM OBSERVATIONS: DOPPLER WINDS \& VARIABILITY OF $\mathrm{SO}_{2}, \mathrm{H}_{2} \mathrm{O}$}

Ground-based submm observations are uniquely suited for study of Venus's mesosphere and lower thermosphere $(70-110 \mathrm{~km})$, with altitude-resolved sensitivity to chemical composition, thermal structure, and Doppler winds above the $\sim 70 \mathrm{~km}$ cloud tops. Altitude resolution is derived from the shape of pressure-broadened submm spectroscopic lines and the exponential variation of pressure with altitude. Submm observations complement the altitudes probed with ground-based IR observations, which probe levels within and below the clouds.

Discoveries supported by ground-based submm spectroscopy include: (1) extreme time variability of Venus mesospheric water vapor on a global scale (Sandor and Clancy, 2005), subsequently also characterized with submm spectroscopy from Earth-orbit (Gurwell et al., 2007), (2) similarly extreme temporal variation of global $\mathrm{SO}$ and $\mathrm{SO}_{2}$ (Sandor et al., 2010), (3) an $\mathrm{SO}_{\mathrm{x}}$ altitude inversion - more $\mathrm{SO}$ and $\mathrm{SO}_{2}$ above than below $84 \mathrm{~km}$ (Sandor et al., 2007, 2010), subsequently observed at higher spatial resolution from Venus Express (Belyaev et al., 2012) and with ground-based submm interferometry (Encrenaz et al., 2015), (4) first measurements of $\mathrm{ClO}$ in the Venus atmosphere (Sandor and Clancy, 2018), corroborating a long held theoretical explanation for stability of the Venus atmosphere as $\mathrm{CO}_{2}$ (Yung and DeMore, 1982), (5) depletion of $\mathrm{HCl}$ above $\sim 82 \mathrm{~km}$ relative to model predictions, showing that contrary to theoretical expectations the primary carrier of chlorine must be something other than $\mathrm{HCl}$ (Sandor and Clancy, 2012; 2017), (6) observational definition of the Venus dayside mesopause (Clancy et al., 2003), (7) characterization of spatial-temporal variability of thermal and CO 
abundance altitude structures (Clancy et al., 2012a), (8) characterization of lower thermospheric $(100-110 \mathrm{~km})$ wind fields that sometimes display retrograde zonal flow (comparable to that seen in the clouds at $70 \mathrm{~km}$ and below), and sometimes present subsolar-to-antisolar (SSAS) axisymmetric flow characteristic of much higher $(150 \mathrm{~km})$ altitudes in the thermosphere (Clancy et al., 2012b). Finally, (9) cross-terminator winds (connecting the quite different dayside vs nightside flow regimes) are distinctly supersonic (Clancy et al., 2015) and display temporal variations on timescales of hours.

The outstanding issue we continue to address is the advancement in understanding of Venus photochemistry, atmospheric dynamics, and the relationship between the two. Submm maps of thermal profiles across the nightside characterize variations in vertical transport - on any given pressure surface warm and cold locations correspond to areas of adiabatic descent and ascent, respectively. Observations show these thermal distributions to be time-variable, on timescales ranging from hours to years, and the relevant goal is to understand what drives these variations. We also seek to understand what drives the extreme temporal variations of water vapor and $\mathrm{SO}_{x}$ $\left(\mathrm{SO}_{2}\right.$ and $\left.\mathrm{SO}\right)$. Ongoing research includes investigations of correlations between the respective variations of water and $\mathrm{SO}_{x}$, which might provide clues regarding each one's variability. Important gas phase chemical reactions between water and these sulfur compounds are known; observational data to demonstrate their chemistry and evident variations is still needed. Another open question is the possible relationship between sulfur/water chemistry and atmospheric dynamics (i.e., possible association between areas of upward/downward transport with abundances of water and sulfur compounds).

\subsection{MID-INFRARED OBSERVATIONS: EVIDENCE OF MOUNTAIN WAVES AND PLANETARY SCALE WAVES}

Ground-based observations at thermal infrared wavelengths are powerful tools to study gravity waves near the cloud tops and planetary scale waves. Observations at $8-12 \mu \mathrm{m}$ with Subaru/COMICS in 2005 and 2007 revealed, for the first time, existence of multiple wave signatures (both patch-like and elongated features) in the night hemisphere of Venus at the cloud top level (Sato et al., 2014). Sequential observations with $5 \mu \mathrm{m}$ wavelength by SpeX of NASA Infrared Telescope Facility (IRTF) depicted slow motions of some wave signatures in the night side prior to Akatsuki's discovery of stationary waves (Fukuhara et al., 2017). The finding of the slow atmospheric motion on the nightside from IRTF/SpeX helped to recognize the unexpected characteristics of the stationary wave in Akatsuki observations.

Multi-wavelength observations from the ground can provide information on the phase variation with height of stationary features and planetary scale waves. This is important for understanding the vertical propagation. Ground-based observations can fill Akatsuki/LIR's observation gaps in terms of stationary features that appear at specific local times: Akatsuki/LIR cannot see those features when the pericenter is located on Venus's afternoon side. Weak positive correlation is 
found between the brightness temperature from LIR and the radiance in UV $(283,365 \mathrm{~nm})$. This suggests that vertical transport of trace gases occurs when the cloud height is changed by vertical winds associated with waves and/or convection. Coordinated cloud and trace gas observations using Akatsuki's LIR and UVI images and ground-based near-IR spectroscopy would be useful for understanding vertical transport and comparing observations to GCM results.

\subsection{COMPOSITION AND TEMPERATURE}

Night side infrared windows, especially near 1.74 and $2.3 \mu \mathrm{m}$, allow for extensive trace gases measurements below the clouds: $\mathrm{CO}, \mathrm{OCS}, \mathrm{SO}_{2}, \mathrm{H}_{2} \mathrm{O}$ (and its isotope $\mathrm{HDO}$ ), $\mathrm{HCl}$ and $\mathrm{HF}$. Understanding the spatial and temporal variability of these species is of paramount importance to constrain deep atmospheric circulation, gas-phase thermochemistry and surface-atmosphere interactions (including possible volcanism). High resolution spectroscopic observations (e.g., using the IRTF/iSHELL spectrometer) decouples spectroscopic degeneracy between trace species by resolving individual absorption lines. With improved high-pressure cross sections for these species, radiative transfer models will be able to retrieve trace gas distributions and temperature profiles between the surface and the cloud base.

\subsection{AIRGLOW, AURORA AND WINDS IN THE TRANSITION REGION}

Airglow emissions occurring in Venus' upper atmosphere are ideal tracers of the atmospheric dynamics at high altitudes. In particular, the UV Nitric Oxide (NO) nightglow peaking at about $110 \mathrm{~km}$ of altitude and the $1.27 \mu \mathrm{m} \mathrm{O}_{2}{ }^{1} \Delta$ airglow peaking around $95 \mathrm{~km}$ of altitude are at the convergence of two circulation regimes: a retrograde super-rotating zonal (RSZ) circulation below $\sim 100 \mathrm{~km}$ and a sub-solar/anti-solar circulation (SSAS) above $100 \mathrm{~km}$ altitude. The fact that these two emission sources appear near midnight and $2 \mathrm{AM}$ (for $\mathrm{O}_{2}{ }^{1} \Delta$ and NO, respectively) reflects how these two sources probe the RSZ to SSAS transition region.
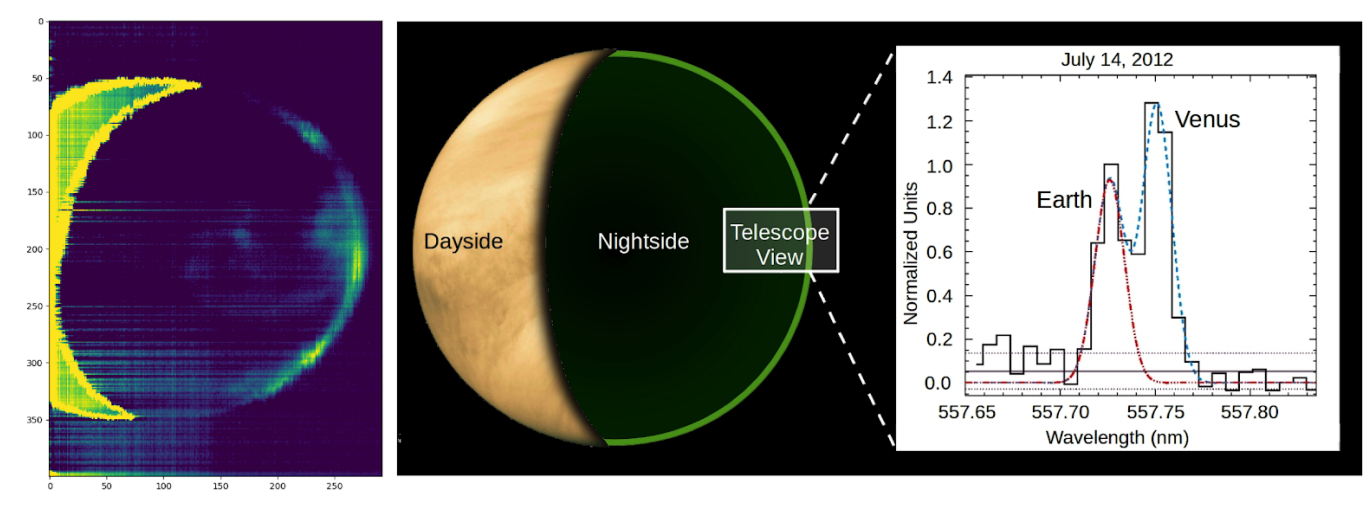

Fig. 2. Left: A slice at $\lambda=1.269 \mu \mathrm{m}$ from an IRTF-SpeX spectral cube (Sept 16, 2007) showing $\mathrm{O}_{2}{ }^{1} \Delta$ airglow at the limb and nightside hemisphere. Right: OI $557.7 \mathrm{~nm}$ auroral emission observed from ARCES on the 3.5m ARC telescope at APO after a CME impact (Gray et al. 2014). 
While Venus lacks an intrinsic magnetic field it does exhibit a unique, diffuse aurora, visible across the nightside of the planet, present after intense solar particles storms such as coronal mass ejections (CMEs), solar flares, and dense solar wind streams (Gray et al. 2014). The OI (1S - 1D) emission at $557.7 \mathrm{~nm}$ (oxygen green line) is readily observable from the ground with a high resolution spectrograph (high enough to separate the Venusian Doppler shifted auroral lines from the terrestrial emission lines, Fig. 2). The green aurora (hypothesized to be a proton aurora) has been detected from APO with ARCES (R 32,000, Gray et al. 2014) and Keck I with HIRES ( $\mathrm{R} \sim 40,000$ mode, Slanger et al. 2001). The OI (3S - 3P) and (5S -3P) auroral lines at 130.4 and $135.6 \mathrm{~nm}$ respectively, were detected from Pioneer Venus Orbiter during an intense CME.

\section{RECOMMENDATIONS FOR IMPROVED GROUND-BASED CAPABILITIES}

\subsection{ADAPTIVE OPTICS FOR EXTENDED OBJECTS}

Venus's angular diameter is about 20" at quadrature and up to 60" at inferior conjunction. Adaptive optics systems regularly achieve spatial resolutions of 50 mas (point spread function's full-width at half-maximum), but few AO systems work on Venus. If they could, resolutions on Venus would be $\sim 30 \mathrm{~km}$. For comparison, Akatsuki's IR2 camera achieved resolutions of $\sim 18$ to $\sim 40 \mathrm{~km}$, depending on its distance from Venus. Venus cannot be corrected with most AO systems, but current solar telescope AO systems work on extended objects and, with multi-conjugate adaptive optics systems, correct fields of view larger than 50". We recommend supporting the adaptation of AO systems to provide corrected imaging of Venus.

\subsection{MID-INFRARED CAMERAS ON LARGE TELESCOPES}

One of the disturbing trends at large telescopes is the decommissioning of mid-infrared instruments (with sensitivities between 5-20 $\mu \mathrm{m}$ ). At the same time, Akatuki's LIR camera (sensitive from $8-12 \mu \mathrm{m}$ ) is responsible for some of Akatsuki's most important discoveries: planetary scale waves (at 3.9 and 5.3-d periods) and stationary features (due to waves launched from mountains). We recommend supporting mid-infrared cameras to make regular observations of Venus between 5 - $20 \mu \mathrm{m}$.

\subsection{IMPROVED CROSS SECTIONS FOR RADIATIVE TRANSFER MODELING}

For spectral modeling of Venus observations, there is a need for laboratory cross sections at high temperatures and pressures. In particular, there is a need for $\mathrm{CO}_{2}$ and $\mathrm{H}_{2} \mathrm{O}$ cross sections at pressures above 10 bar. These cross sections are difficult to measure, but techniques like cavity ringdown and photoacoustic spectroscopy can be applied. We recommend support of laboratory studies to measure cross sections at high pressures and temperatures. These cross sections are important for accurate retrievals of trace gases and temperature profiles in Venus's lower atmosphere. 


\section{REFERENCES}

Asay-Davies, X. S. et al. (2009), Jupiter's shrinking Great Red Spot and steady Oval BA: Velocity measurements with the 'Advection Corrected Correlation Image Velocimetry' automated cloud-tracking method. DOI:10.1016/j.icarus.2009.05.001

Fukuhara, T. et al. (2017), Large stationary gravity wave in the atmosphere of Venus. DOI:10.1038/ngeo2873

Gray, C. et al. (2014), The effect of solar flares, coronal mass ejections, and solar wind streams on Venus' $5577 \AA$ A oxygen green line. DOI:10.1016/j.icarus.2014.01.029

Horinouchi, T. et al. (2020), How waves and turbulence maintain the super-rotation of Venus' atmosphere. DOI:10.1126/science.aaz4439

Machado, P. et al. (2017), Venus cloud-tracked and doppler velocimetry winds from CFHT/ESPaDOnS and Venus Express/VIRTIS in April 2014. DOI:10.1016/j.icarus.2016.12.017

Sato, T. M. et al. (2014), Cloud top structure of Venus revealed by Subaru/COMICS mid-infrared images. DOI:10.1016/j.icarus.2014.09.004

Slanger, T. et al. (2001), Discovery of the Atomic Oxygen Green Line in the Venus Night Airglow. DOI:10.1126/science.291.5503.463

Belyaev et al. (2011), Vertical Profiling of SO2 and SO above Venus' Clouds by SPICAV/SOIR Solar Occultations. doi:10. 1016/j.icarus.2011.09.025.

Clancy, R.T., B.J. Sandor, and G.H. Moriarty-Schieven, Observational Definition of the Venus Mesopause: Vertical Structure, Diurnal Variation, and Short-term Instability,doi:10.1016/S0019-1035(02)00022-2.

Clancy, R.T., B.J. Sandor, and G.H. Moriarty-Schieven (2012a). Thermal Structure and CO Distri- bution for the Venus Mesosphere/Lower Thermosphere: 2000-2009 Inferior Conjunction Sub-millimeter CO Absorption Line Observations. doi:10.1016/j.icarus.2011.05.032.

Clancy, R.T., B.J. Sandor, and G.H. Moriarty-Schieven (2011),. Circulation of the Venus Upper Mesosphere/Lower Thermosphere: Doppler Wind Measurements from 2001-2009 Inferior Conjunction, Submillimeter CO Absorption Line Observations. doi:10.1016/j.icarus.2011.05.021.

Clancy, R.T., B.J. Sandor, and J. Hoge (2015),. Doppler Winds Mapped around the Lower Thermospheric terminator of Venus: 2012 Solar Transit Observations from the James Clerk Maxwell Telescope. http://dx.doi.org/10.1016/j.icarus.2015.03.031

Encrenaz, T., R. Moreno, A. Moullet, E. Lellouch, and T. Fouchet (2015). Submillimeter mapping of mesospheric minor species on Venus with ALMA. https://doi.org/10.1016/j.pss.2015.01.011.

Gurwell, M.A, Melnick, G.J., Tolls, V., Bergin, E.A., Patten, B.M. (2006), SWAS observations of water vapor in the Venus mesosphere, doi:10. 1016/j.icarus.2006.12.004.

Sandor, B.J., and R.T. Clancy (2005), Water Vapor Variations in the Venus Mesosphere from Microwave Spectra, Icarus, 177., 129-143, 2005. doi:10.1016/j.icarus.2005.03.020.

Sandor, B.J., R.T. Clancy, and G.H. Moriarty-Schieven (2007). SO and SO2 in the Venus Mesosphere: Observations of Extreme and Rapid Variation. 2007 BAAS., v.39, abstract no. 45.08. p.503. 2007.

Sandor, B.J., R.T. Clancy, G.H. Moriarty-Schieven, and F.P. Mills (2010). Sulfur Chemistry in the Venus Mesosphere from SO2 and SO Microwave Spectra. doi:10.1016/j.icarus.2010.02.013.

Sandor, B.J., and R.T. Clancy (2012), Observations of HCl Altitude Dependence and Temporal Variation in the 70-100 km Mesosphere of Venus.http://dx.doi.org/10.1016/j.icarus.2012.05.016.

Sandor, B. J., Clancy, R. T. Diurnal Observations of HCl Altitude Variation in the 70-100 km Mesosphere of Venus. Icarus, 290. 156-161. 2017. https://doi.org/10.1016/j.icarus.2017.02.017

Sandor, B.J., and R.T. Clancy (2018), First measurements of $\mathrm{ClO}$ in the Venus atmosphere - Altitude dependence and temporal variation. https://doi.org/10.1016/j.icarus.2018.04.022

Yung, Y.L., and W.B. DeMore, Photochemistry of the stratosphere of Venus: Implications for atmospheric evolution, Icarus 51, 199-207. 1982. https://doi.org/10.1016/0019-1035(82)90080-X 